\title{
Mortalidad Perinatal y Materna
}

\author{
Dr. Alberto Moreno Rojas*
}

\section{INTRODUCCION}

En el Hospital General de Neiva, desde su inauguración, hace aproximadamente 13 años, no ha tenido lugar una revisión de los servicios que presta en todas las especialidades médicas y aunque cuenta con una dotación hospitalaria aceptable para realizar diagnósticos y tratamientos adecuados a los pacientes que allí consultan, así como una nómina de médicos generales, especialistas y personal de enfermería con un buen nivel académico, se desconoce el resultado médico de la actividad profesional en las diferentes especialidades.

Conocedor que en la actualidad la demanda de servicios en nuestro hospital ha aumentado en un considerable porcentaje anual debido, posiblemente, a la calidad de servicios que se prestan, a la facilidad de transporte de regiones vecinas, al moderado costo de estos servicios comparados con otras instituciones, al desplazamiento de la población rural a la ciưdad y a la atención a los sectores más desprotegidos de la comunidad local, se hace necesario conocer la realidad médica de la situación hospitalaria por quienes laboramos en él para lograr unos mejores resultados.

Preocupado por la falta de un estudio de morbi-mortalidad perinatal y sabiendo que el estudio de la patología materna y feto-neonatal es indispensable en todo servicio obstétrico, pues su desconocimiento impide la realización de cambios o refuer-

Médico Ginecobstetra Hospital General de Neiva. zos de conductas médicas que lleven a la solución de los problemas maternos y fetales, (3), (6), (2), practiqué la revisión de $\mathrm{H}$. C. obstétricas y neonatales, durante el período de 1 año, de pacientes atendidas en nuestra institución.

\section{OBJETIVOS}

El objetivo fundamental de éste trabajo es conocer la tasa de mortalidad perinatal y materna en el Hospital General de Neiva, para lo cual se revisaron todas las H. C. (2.437) de pacientes atendidas durante el período comprendido entre el 1 de junio de 1987 y el 31 de mayo de 1988.

También analizar las tasas de mortalidad perinatal y materna y relacionarlas con las variables conocidas e investigadas para, de acuerdo con su resultado, tomar las medidas del caso que nos lleven a prestar un mejor servicio y obtener, desde luego, mayores beneficios para nuestra comunidad.

\section{METODOLOGIA}

Para realizar el presente estudio retrospectivo se revisaron todas las $\mathrm{H}$. C. de pacientes atendidas en el servicio de Ginecobstetricia en la sección obstétrica durante el período de 1 año comprendido desde el 1 de junio de 1987 hasta el 31 de mayo de 1988 .

Se atendieron 2.437 pacientes que dieron a luz 2.445 productos y sus historias se revisaron ma- 
nualmente, pues no tenemos en la institución servicio de sistematización para las H.C. Se incluyeron variables como edad, estado civil, grado de escolaridad, procedencia o residencia habitual cuando tenían menos de 6 meses de residir en Neiva, control prenatal, tipo de parto, complicaciones en el embarazo y parto, datos del R.N., y resultado final de éste. Se incluyeron todos los embarazos sin tener en cuenta la vía de terminación del parto; se tomaron todos los R.N. con peso de 500 gms o más y solamente se excluyeron los abortos y los embarazos ectópicos.

Para efectos estadísticos relacionados con las tasas de mortalidad perinatal solamente se incluyeron los fetos que pesaron 1.000 gms o más al nacer.

La falta de sistematización, así como también la falla en la elaboración de la H. C. hizo que algunas variables no se hubieran podido investigar por falta de datos y también por pérdida de partes del compendio de la historia de pacientes tratadas.

\section{Definición de términos}

Embarazo a término: es el comprendido entre el final de la semana 37 y el final de la semana 42 (de 259 a 293 días completos ).

Embarazo pretérmino: es aquel que dura menos de 37 semanas completas (menos de 259 días), desde el primer día de la última menstruación.

Bajo peso al nacer: todo R.N. con más de 500 gms y menos de 2.500 gms de peso, independiente de la edad gestacional.

Retardo Crecimiento Intrauterino: 'todo R.N. con más de 37 semanas de gestación confiable y peso inferior a $2.500 \mathrm{gms}$ (pequeño para la edad gestacional).

Mortalidad Perinatal: toda muerte del producto ocurrida a partir de la semana 28 de gestación y hasta el 7o. día de nacido.

Muerte Materna: es aquella que ocurre durante el embarazo y hasta la finalización del puerperio, independiente de la edad gestacional y por cualquier causa relacionada o agravada por el embarazo y su manejo. Puede ser de causa directa o indirecta.

\section{RESULTADOS}

Se revisaron 2.437 historias clínicas de pacientes obstétricas atendidas y se obtuvieron 2.445 productos, contando 6 partos gemelares y 1 de trillizos.
El estado civil de las pacientes atendidas correspondió en un poco más de la mitad a las casadas con el $51 \%$ y el restante $49 \%$ se encuentra repartido entre unión libre y solteras. Cuadro 1.

CUADRO 1

ESTADO CIVIL

\begin{tabular}{|lcc|}
\hline & $\%$ & Número \\
\hline Casadas & 51.0 & 1.242 \\
U. Libre & 21.0 & 511 \\
Solteras & 28.0 & 684 \\
Total & 100.0 & 2.437 \\
\hline
\end{tabular}

En el cuadro 2, podemos observar que solamente el $75.7 \%$ de las pacientes viven en Neiva; las demás son de poblaciones vecinas y departamentos limítrofes.

\section{CUADRO 2}

RESIDENCIA

\begin{tabular}{|ccc|}
\hline & $\%$ & Número \\
\hline Neiva & 75.7 & 1.846 \\
\hline
\end{tabular}

En el Cuadro 3, encontramos los grupos de edad materna donde llama la atención el número de adolescentes gestantes (menores de 15 y 15 - 19) que corresponde a 596 pacientes (24.4\%); los grupos de edad entre los 15 - 29 años constituyen el $81.3 \%$ (1985 pacientes) y se observa el franco predominio en el grupo de pacientès entre 20 - 24 años. Después de los 30 años de edad la incidencia de gestaciones se reduce en forma notoria.

CUADRO 3

EDAD MATERNA

\begin{tabular}{|ccc|}
\hline & $\%$ & Número \\
\hline$<\quad 15$ & 0.50 & 12 \\
$15-19$ & 24.0 & 584 \\
$20-24$ & 36.8 & 900 \\
$25-29$ & 20.5 & 501 \\
$30-34$ & 10.3 & 250 \\
$35-39$ & 5.8 & 140 \\
$40-44$ & 2.1 & 50 \\
Total & 100.0 & 2.437 \\
\hline
\end{tabular}


En la distribución de la paridad encontramos el mayor número de las pacientes en las primigrávidas con 914 casos (37.5\%). Llama la atención el grupo de 7 y más partos con una cifra de 117 casos $(4.8 \%)$. Cuadro 4 .

CUADRO 4

DISTRIBUCION DE LA PARIDAD

\begin{tabular}{|ccc|}
\hline & $\%$ & Número \\
\hline 1 & 37.5 & 914 \\
2 & 23.8 & 580 \\
3 & 16.5 & 402 \\
4 & 8.6 & 210 \\
5 & 6.0 & 146 \\
6 & 2.8 & 68 \\
$7 y+$ & 4.8 & 117 \\
Total & 100.0 & 2.437 \\
\hline
\end{tabular}

De las pacientes atendidas en la institución solamente 117 (4.80\%) tuvieron control prenatal.Cuadro 5 .

CUADRO 5

CONTROL PRENATAL

\begin{tabular}{|lll|}
\hline & $\%$ & Número \\
\hline Pacientes & 4.80 & 117 \\
\hline
\end{tabular}

En el Cuadro 6, observamos la edad gestacional al momento del parto, donde el promedio de gestación es de 40 semanas (91.8\%); Pretérmino del $7.7 \%$. La incidencia de postmaduros por amenorrea es solamente de 8 pacientes $(0.33 \%)$.

CUADRO 6

EDAD GESTACIONAL AL PARTO (Semanas)

\begin{tabular}{|crc|}
\hline & \multicolumn{1}{c|}{$\%$} & Número \\
\hline $20-28$ & 1.32 & 32 \\
$29-36$ & 6.47 & 157 \\
$37-42$ & 91.88 & 2.240 \\
+42 & 0.33 & 8 \\
Total & 100.00 & 2.437 \\
\hline
\end{tabular}

El puntaje de APGAR, Cuadro 7, nos mostró que al minuto de nacido hubo 721 casos $(29.48 \%$ ) clasificados entre 6 y menos y a los cinco minutos fueron 494 casos $(20.2 \%)$. Con puntaje de 7 y más encontramos 1.724 casos al minuto $(70.52 \%)$ y 1.951 casos a los cinco minutos $(79.80 \%)$.

\section{CUADRO 7}

\section{PUNTAJE DE APGAR}

\begin{tabular}{|c|c|c|c|c|c|}
\hline \multicolumn{3}{|c|}{ Un minuto } & & \multicolumn{2}{|c|}{ Cinco minutos } \\
\hline & $\%$ & Número & & $\%$ & Número \\
\hline 0 & 8.43 & 206 & 0 & 4.04 & 99 \\
\hline $1-3$ & 5.27 & 129 & $1-3$ & 6.06 & 148 \\
\hline $4-6$ & 15.78 & 386 & $4-6$ & 10.10 & 247 \\
\hline $7-9$ & 70.52 & 1.724 & $7-9$ & 79.80 & 1.951 \\
\hline Total & 100.00 & 2.445 & & 100.00 & 2.445 \\
\hline
\end{tabular}

En el Cuadro 8, peso en $g$ al nacer, encontramos que 180 R. N. pesaron menos de $2.500 \mathrm{~g}$ $(7.35 \%)$. El grupo comprendido entre 2.500 y 3.500 g mostró una cifra de 1.403 R. N. (57.3\%). Los pesos superiores a $4.500 \mathrm{~g}$ constituyeron el $1.43 \%$.

CUADRO 8

DISTRIBUCION DE PESO AL NACER (gms.)

\begin{tabular}{|ccc|}
\hline & $\%$ & Número \\
\hline $500-999$ & 0.69 & 17 \\
$1000-1499$ & 0.89 & 22 \\
$1500-1999$ & 1.67 & 41 \\
$2000-2499$ & 4.10 & 100 \\
$2500-2999$ & 20.04 & 490 \\
$3000-3499$ & 37.35 & 913 \\
$3500-3999$ & 27.50 & 672 \\
$4000-4499$ & 6.33 & 155 \\
4500 y & 1.43 & 35 \\
Total & & \\
& 100.00 & 2.445 \\
\hline
\end{tabular}

En el análisis de las muertes perinatales encontramos lo siguiente: Mortalidad Total: 146 casos. Mortalidad Depurada: 129 casos. Se excluyeron 17 R. N. que pesaron menos de $1.000 \mathrm{~g}$, según recomendaciones del CLAP para fines estadísticos. 
Se clasificó el momento de la muerte, Cuadro 9, como anteparto con el $34.9 \%$ (45 casos); intraparto con el $32.6 \%$ (42 casos), neonatal temprano con el $26.3 \%$ (34 casos) y neonatal tardío con el $6.2 \%$ ( 8 casos).

CUADRO 9

MOMENTO DE LA MUERTE

\begin{tabular}{|lrc|}
\hline & $\%$ & Número \\
\hline Anteparto & 34.9 & 45 \\
Intraparto & 32.6 & 42 \\
Neonatal temp. & 26.3 & 34 \\
Neonatal tardío & 6.2 & 8 \\
Total & 100.0 & 129 \\
\hline
\end{tabular}

De acuerdo con los grupos de edad materna, Cuadro 10, los comprendidos entre $20-29$ años presentaron índice de mortalidad del $54.3 \%$. La mortalidad fue baja en los grupos de edades extremas.

CUADRO 10

GRUPOS DE EDAD MATERNA

\begin{tabular}{|crr|}
\hline & \multicolumn{1}{c|}{$\%$} & Número \\
\hline$<15$ & 1.60 & 2 \\
$15-19$ & 15.50 & 20 \\
$20-24$ & 25.60 & 33 \\
$25-29$ & 28.70 & 37 \\
$30-34$ & 10.80 & 14 \\
$35-39$ & 13.20 & 17 \\
$40-44$ & 4.60 & 6 \\
Total & 100.00 & 129 \\
\hline
\end{tabular}

Los nacidos con edad gestacional menor de 36 semanas son $68(42,7 \%)$ y el resto, 61 pacientes, $(47.3 \%)$ tenían entre 37 y 42 semanas de gestación. Cuadro 11.

CUADRO 11

SEMANAS DE GESTACION

\begin{tabular}{|lcc|}
\hline & $\%$ & Número \\
\hline $20-28$ & 17.8 & 23 \\
$29-36$ & 34.9 & 45 \\
$37-42$ & 47.3 & 61 \\
+42 & & 129 \\
Total & 100.0 & \\
\hline
\end{tabular}

En el Cuadro 12, observamos que los pesos de los R. N. de menos de $2.500 \mathrm{~g}$ corresponden a 70 pacientes $(54.3 \%$ ) y el grupo de 2.500 a $3.500 \mathrm{~g}$ es de 41 pacientes $(31.8 \%)$. Las necropsias practicadas fueron solamente en 12 casos, $(9.30 \%)$.

CUADRO 12

PESO AL NACER (GMS)

\begin{tabular}{|ccc|}
\hline & $\%$ & Número \\
\hline $1000-1499$ & 15.5 & 20 \\
$1500-1999$ & 22.5 & 29 \\
$2000-2499$ & 16.3 & 21 \\
$2500-2999$ & 18.6 & 24 \\
$3000-3499$ & 13.2 & 17 \\
$3500-3999$ & 8.5 & 11 \\
$4000-4499$ & 3.9 & 5 \\
$4500 y+$ & 1.5 & 2 \\
& & 129 \\
\hline
\end{tabular}

La tasa de mortalidad perinatal se calculó en $49.48 \times 1.000$ nacidos vivos.

Las muertes maternas directas, Cuadro 13, correspondieron en total a 10 pacientes, distribuidas así: Sepsis 4, Toxemia 3 y Hemorragia 3.

$$
\text { CUADRO } 13
$$

MORTALIDAD MATERNA

\begin{tabular}{|lcc|}
\hline & $\%$ & Número \\
\hline Toxemia & 30.0 & 3 \\
Hemorragia & 30.0 & 3 \\
Sepsis & 40.0 & 4 \\
Total & 100.0 & 10 \\
\hline
\end{tabular}

La tasa de mortalidad materna se calculó en $40.8 \times 10.000$ nacidos vivos.

\section{COMENTARIOS}

Uno de los mayores problemas en nuestro medio y como lo muestra el prescnte estudio, es la elevada tasa de mortalidad perinatal y materna en el Hospital General de Neiva.

De acuerdo con algunos autores quienes afirman que la eficiencia de la atención perinatal se mi- 
de por las bajas tasas de mortalidad materna y perinatal (1), (4), (6), conocedores de la situación perinatal en nuestro medio, teniendo la infraestructura necesaria en nuestra institución y que las principales causas de mortalidad presentadas son factibles de reducir y controlar, todos nuestros esfuerzos deben encaminarse a lograr tan noble finalidad que redundará en beneficios médicos y para la comunidad.

El profesor Fernando Sánchez T., dice en su libro de Alto Riesgo Obstétrico: “. . . . . que la inversión que se haga en este campo se verá compensada con creces, pues con ella se va a mejorar la calidad de vida de los nuevos ciudadanos" (5). En la revisión presentada por Uriza y Barragán (7) comentan que hay estudios que afirman que para los paises del tercer mundo en vías de desarrollo o subdesarrollados, es mucho más importante un adecuado y cuidadoso control prenatal para reducir la tasa de mortalidad perinatal, que lo que se puede lograr con las costosas y sofisticadas unidades de Cuidado Intensivo Neonatal, que viene a agravar la ya precaria situación económica de las instituciones hospitalarias.

\section{CONCLUSIONES}

La tasa de mortalidad perinatal es de $49.4 .8 \times$ 1.000 nacidos vivos.

La tasa de mortalidad materna es de $40.8 \mathrm{x}$ 10.000 nacidos vivos.

El índice de bajo peso al nacer, menos de $2.500 \mathrm{~g}$, es de $7.35 \%$.

El índice de prematuridad es de $7.7 \%$.

La incidencia de cesáreas es de $12.8 \%$.

El porcentaje de muertes en los R. N. con bajo peso es de $38.8 \%$.

El porcentaje de muertes en los R. N. de pretérmino fue de $37.5 \%$.

Se practicaron solamente 12 necropsias en $\mathrm{R}$. N. o sea el $9.3 \%$.

Sólo el $75 \%$ de las pacientes atendidas en la institución viven en Neiva; el resto es de municipios vecinos y departamentos limítrofes.

La principal causa de muerte materna directa fue la sepsis con 4 casos (40\%), luego la hemorragia y la toxemia con 3 casos cada una.
- El grupo formado por pacientes con siete y más partos corresponde principalmente a pacientes de procedencia rural.

- Las causas más frecuentes de mortalidad perinatal encontradas en nuestro estudio se pueden reducir con la aplicación de medidas preventivas disponibles en nuestro medio.

- En nuestro estudio el grupo de edad materna comprendido entre $20-29$ años presenta el mayor índice de nacimientos (1.401) o sea el $57.3 \%$, y así también es el grupo que presenta el mayor índice de mortalidad perinatal (70) o sea $54.3 \%$.

- Debido a fallas en la elaboración de las H. C. por el no llenado de algunos de los formularios o en la elaboración de la epicrisis, no fue posible lograr datos concluyentes sobre el diagnóstico final fetal, lo cual constituía otro de nuestros principales objetivos a investigar.

\section{RECOMENDACIONES}

1. La creación de un comité permanente de mortalidad perinatal, con mentalidad crítica constructiva, el cual debe estar integrado por personal del Dpto. de Ginecobstetricia, Pediatría, Enfermería, Anatomía Patológica y Salubrista.

2. Incrementar la consulta prenatal la cual debe ser de buena calidad y frecuencia, para prevenir principalmente la prematurez y el bajo peso al nacer.

3. Practicar las necropsias a todos los R. N. que hayan fallecido en el período peri-neonatal y así poder conocer a fondo nuestra patología.

\section{RESUMEN}

En el presente estudio se analizan los datos maternos y neonatales de 2.445 partos atendidos en la sección obstétrica del Departamento de Obstetricia y Ginecología del Hospital General de Neiva, durante el período comprendido entre el 1 de junio de 1987 al 31 de mayo de 1988. Se revisan las posibles causas de mortalidad perinatal y materna.

En el trabajo se incluyen todos los embarazos si tener encuenta la vía de terminación del parto. 
Se tomaron todos los R. N. con peso de $500 \mathrm{~g}$ o más; pero para fines estadísticos, según lo ordenado por el CLAP, sólo se tomaron los que pesaron 1.000 g o más. Se excluyeron los abortos y los embarazos ectópicos.

Se atendieron 2.445 partos resultado de 2.437 embarazos, con 6 pares de gemelos y un caso de trillizos. La tasa de mortalidad perinatal encontrada fue de $49.48 \times 1.000$ nacidos vivos. Durante el mismo período murieron 10 madres de causa obstétrica directa.

Se revisan las causas de muerte materna y fetal y se hacen recomendaciones para disminuir en forma notoria las tasas de mortalidad tanto materna como fetal.

\section{BIBLIOGRAFIA}

1. Centro Latinoamericano de Perinatología. Incidencia del bajo peso al nacer y mortalidad perinatal en América Latina. Salud Perinatal 1: 3-6, 1983.

2. DIAZ, J. L. y col. Guías de organización y normatización básica de la asistencia neonatal. CLAP 1028, 1984.

3. HUbIZ, H. A. Morbi-Mortalidad Perinatal. Rev. Col. de Obstetricia y Ginecología. Vol. 35, No. 6, página 440, 1984.

4. Salud Perinatal. Boletín del CLAP. Vol. 2, No. 4, 1985.
5. SANCHEZ, T.F. Alto Riesgo Obstétrico. Ed. UNAL de Colombia, 1988, pág. 19, 1a. Edición.

6. SCHWARCZ, R. y cols. Bajo peso al nacer y Mortalidad Perinatal en Maternidades de América Latina. Ed. por OPS No. $461,1984$.

7. URIZA, G.G.; BARRAGAN, EJ. Mortalidad Perintal. Rev. Col. de Obstetricia y Ginecología, Vol. 37, No. 2, Pág. 91, 1986. 